
transfer RNA release from the E-site

\author{
Christian B. F. Andersen ${ }^{1 \star}$, Thomas Becker ${ }^{2} \dagger$, Michael Blau ${ }^{2}$, Monika Anand ${ }^{3}$, Mario Halic $^{2} \dagger$, Bharvi Balar ${ }^{3}$, \\ Thorsten Mielke ${ }^{4}$, Thomas Boesen ${ }^{1}$, Jan Skov Pedersen ${ }^{5}$, Christian M. T. Spahn ${ }^{6}$, Terri Goss Kinzy ${ }^{3}$, \\ Gregers R. Andersen ${ }^{1} \&$ Roland Beckmann ${ }^{2} \dagger$
}

\begin{abstract}
Elongation factor eEF3 is an ATPase that, in addition to the two canonical factors eEF1A and eEF2, serves an essential function in the translation cycle of fungi. eEF3 is required for the binding of the aminoacyl-tRNA-eEF1A-GTP ternary complex to the ribosomal A-site and has been suggested to facilitate the clearance of deacyl-tRNA from the E-site. Here we present the crystal structure of Saccharomyces cerevisiae eEF3, showing that it consists of an amino-terminal HEAT repeat domain, followed by a four-helix bundle and two ABC-type ATPase domains, with a chromodomain inserted in ABC2. Moreover, we present the cryo-electron microscopy structure of the ATP-bound form of eEF3 in complex with the post-translocational-state $80 \mathrm{~S}$ ribosome from yeast. eEF3 uses an entirely new factor binding site near the ribosomal E-site, with the chromodomain likely to stabilize the ribosomal L1 stalk in an open conformation, thus allowing tRNA release.
\end{abstract}

Protein synthesis requires, in general, only two canonical GTPase elongation factors. eEF1A (known as EF-Tu in prokaryotes) recruits cognate aminoacyl-tRNAs to the A-site of the ribosome, and, after peptidyl transfer, eEF2 (EF-G in prokaryotes) catalyses translocation of the messenger RNA and the transfer RNAs from the A- and P-sites to the P- and E-sites. In contrast to the canonical factors, eEF3 has an ATPase activity that is stimulated by ribosomes. It interacts with both ribosomal subunits ${ }^{1-3}$, competes with eEF2 for binding to ribosomes, and stimulates eEF1A-dependent binding of cognate aminoacyltRNA to the ribosomal A-site ${ }^{1,4}$. Because, according to the allosteric three-site model of the ribosomal elongation cycle, E-site release is required for efficient A-site binding, it has been suggested that eEF3 functions as a so-called 'E-site factor ${ }^{\text {'4,5 }}$. Moreover, ATP hydrolysis by eEF3 is required in every elongation cycle to allow chasing of deacyltRNA from the E-site 4 .

eEF3 belongs to the family of ABC (ATP-binding cassette) proteins that includes proteins involved in transport across membranes, DNA repair, and translation. The membrane proteins of this class especially represent important targets for development of novel therapeutic strategies. The proteins contain ATP/ADP-binding $\mathrm{ABC}$ domains, which convert chemical energy derived from binding of ATP or its hydrolysis into a 'powerstroke' of mechanical energy ${ }^{6}$. $\mathrm{ABC}$ proteins function as either homodimers or as twin-cassette proteins with two $\mathrm{ABC}$ domains within the same polypeptide.

The ribosome exhibits very dynamic behaviour, such as the ratchet movement ${ }^{7}$ or the movement of the L1 and the L7/L12 stalks ${ }^{8-11}$. Hence, an intriguing question is how the interaction of eEF3 with the ribosome is correlated with its dynamic properties as an $\mathrm{ABC}$ protein, and how the energy derived from binding/hydrolysis of ATP is used for its function.

\section{Crystal structure of eEF3}

Three crystal structures of residues 1-980 of eEF3 in the apo state $(2.7 \AA)$, in complex with ADP $(2.4 \AA)$, or in complex with the nonhydrolysable ATP analogue ADPNP (3.0 A) were solved (Supplementary Table 1) and they have virtually the same overall conformation. The deletion of residues 981-1,044 does not alter the ability of the protein to function as the only form of eEF $3^{12}$. The protein has five structural domains (Fig. 1a, b and Supplementary Fig. 1). Residues 1-321 are organized in classical HEAT repeats ${ }^{13}$ with a total of 16 helices. Instead of forming a single long, curved structure as found in PP2 $\mathrm{A}^{14}$, the HEAT domain of eEF3 makes a sharp bend after the first 10 helices. The HEAT domain is connected through a flexible linker to a four-helix bundle (4HB domain; residues 336-413), which packs against the bottom of the concave face of the HEAT domain.

The remaining residues of eEF3 are organized into $\mathrm{ABC} 1$ and $\mathrm{ABC} 2$ domains homologous to the nucleotide-binding domains of $\mathrm{ABC}$ transporters and DNA repair enzymes ${ }^{15-18}$. These domains each consist of two subdomains (or lobes). Lobe I (residues 418-492 and 563-636 in ABC1, and 637-734 and 915-976 in ABC2; also known as the ATP-binding core) is formed by two $\beta$-sheets that wrap around a central $\alpha$-helix and three exposed helices. Lobe II (493-562 in ABC1, and 734-760 and 870-914 in ABC2; also known as the $\alpha$-helical subdomain) is composed of four helices in $\mathrm{ABC} 1$ and five in $\mathrm{ABC} 2$. The arrangement of the two subdomains in both $A B C 1$ and $A B C 2$ is similar to that found in structures of other $\mathrm{ABC}$ domains in their apo or ADP-bound states with DALI ${ }^{19} Z$-scores of $13-19$, and the $Z$-score of eEF3 $\mathrm{ABC} 1$ versus $\mathrm{ABC} 2$ is 15 . Hence, the two cassettes in eEF3 are not more similar to each other than to other ABC proteins.

The chromodomain (residues 761-869) is an insert within the $\alpha$-helical subdomain of ABC2 and contains a five-stranded $\beta$-sheet 
traversed by an $\alpha$-helix. Homologous domains from nuclear proteins interact with histones, DNA and $\mathrm{RNA}^{20}$. The $\mathrm{ABC} 1$ domain has a limited interface to other domains, whereas the $\mathrm{ABC} 2$ domain interacts with the $4 \mathrm{HB}$ domain and the $\mathrm{N}$-terminal part of the HEAT domain (Fig. 1a, b). The chromodomain is located at the convex face of the HEAT domain, but makes only a few contacts with it.

In both the ADP and the ADPNP complexes of eEF3, the nucleotide was found $14 \AA$ away from its expected location towards the H-loop of $\mathrm{ABC} 2$ (Fig. 1c). Binding to the canonical site is prevented by its overlap with Phe 35 from the HEAT domain (Supplementary Fig. 2). In an attempt to disrupt the adeninebinding pocket, we mutated the non-conserved (see Supplementary Fig. 3) Glu 42 to alanine and Ser 82 to tryptophan. These mutations had little effect on yeast growth (data not shown), questioning the physiological relevance of the novel mode of nucleotide binding in
$\mathrm{ABC}$. No nucleotide is bound in the $\mathrm{ABC} 1$ domain, but a single sulphate ion is bound to its P-loop mimicking the $\alpha$ - and $\beta$-phosphates. A second sulphate mimics $\gamma$-phosphate at the positive helix dipole of the signature motif (Supplementary Figs 1 and 2c), the hallmark of the ABC superfamily. This confirms the previous suggestion that the signature motif binds the inorganic phosphate released by ATP hydrolysis ${ }^{16}$.

\section{SAXS analysis of eEF3}

Compared with the ATP-bound homodimer structures of MJ0796 and Rad50, the ABC2 domain in eEF3 is rotated approximately $120^{\circ}$ away from $\mathrm{ABC} 1$. To investigate whether the functionally important $\mathrm{ABC}$ tandem formation inferred from other $\mathrm{ABC}$ protein structures also takes place in eEF3, we created a model of the ATP state in solution. We maintained the overall location of $\mathrm{ABC} 1$ and placed
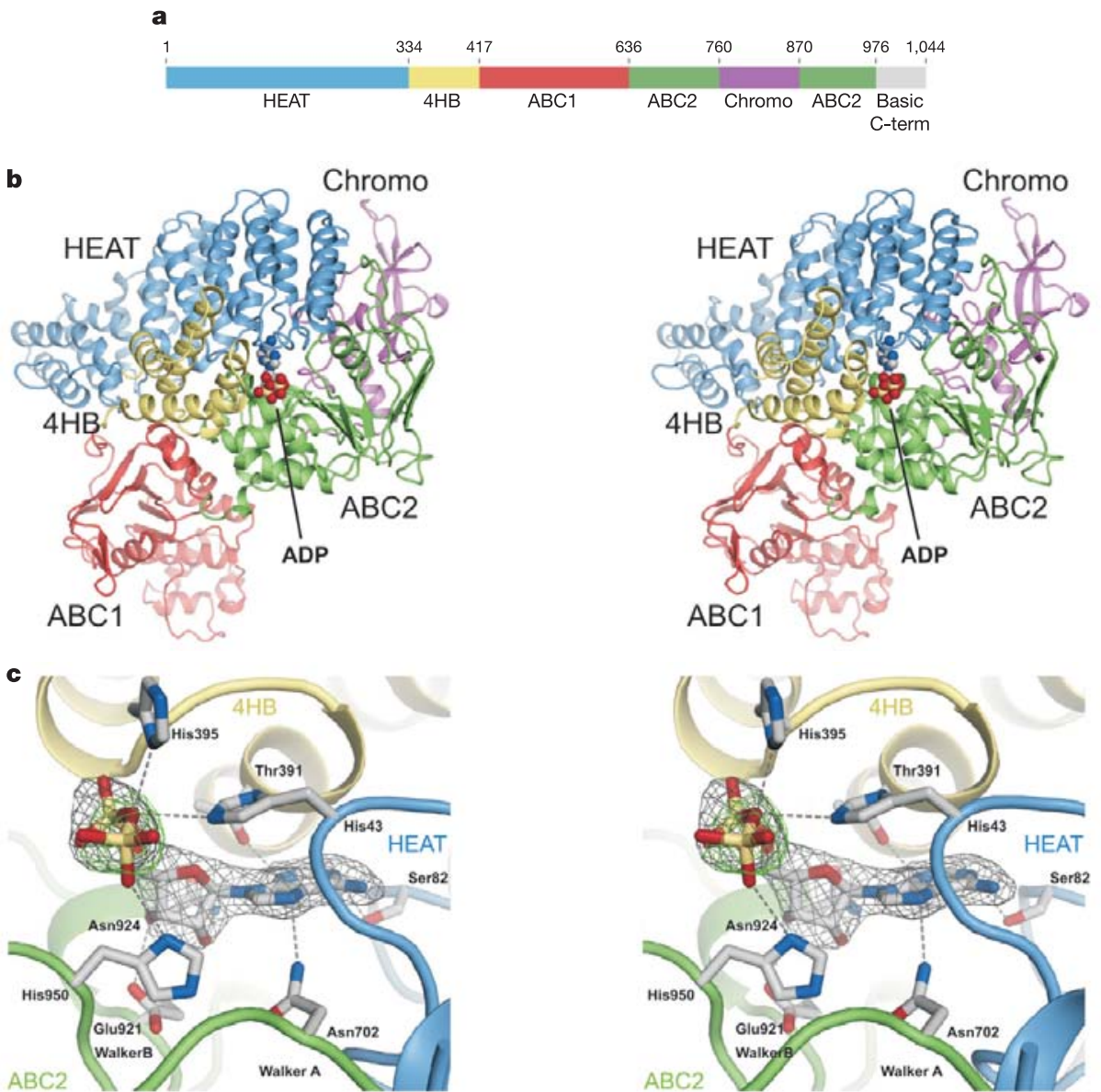

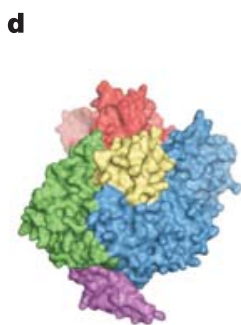

Crystal structure

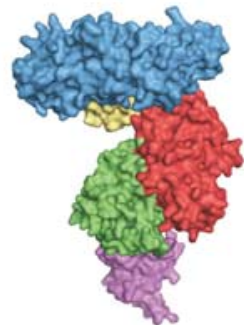

ATP model

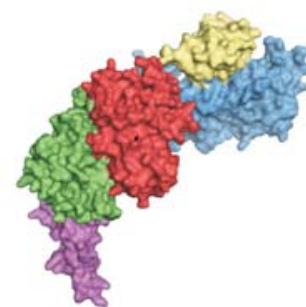

Cryo-EM structure
Figure 1 Structures of Saccharomyces cerevisiae eEF3 and nucleotide binding. a, Schematic representation of the eEF3 sequence. See the text for a description of the different domains. Chromo, chromodomain; C-term, carboxy-terminal domain. $\mathbf{b}$, Stereo view of the crystal structure of eEF3ADP. The nucleotide is shown in ball-and-stick representation. c, Stereo view of the nucleotide-binding site. The electron density of ADP is generated from an omit map contoured at $1.5 \sigma$ (grey) or at $0.8 \sigma$ around the $\beta$-phosphate (green). d, Three different conformations of eEF3. Left: crystal structure of eEF3. Middle: ATP model of eEF3 constructed using homology to the structure of MJ0796. Right: Cryo-electron microscopy (cryo-EM) reconstruction of eEF3 on the $80 \mathrm{~S}$ ribosome. 
ABC2 according to the ATP-induced dimer of MJ0796 ${ }^{16}$. This was then followed by the rearrangement of the relative location of the two subdomains in both $\mathrm{ABC}$ domains (Fig. 1d). This results in an elongated molecule with the chromodomain located at the tip, which, in contrast to the crystal structure, is in good agreement with small-angle X-ray scattering (SAXS) data collected at $\mathrm{pH} 7.2$ (Supplementary Fig. 4). SAXS data collected at the crystallization $\mathrm{pH}$ of 5.2 indicate a more compact conformation of eEF3. Hence, in solution a conformational change within eEF3 can be induced by lowering the $\mathrm{pH}$; however, the physiological relevance of this flexibility is unknown.

\section{Reconstitution and cryo-EM of the eEF3-ribosome complex}

Purified eEF3 was bound to empty ribosomes or to programmed ribosome nascent chain complexes (RNCs) in the post-translocational (post) state, bearing a peptidyl-tRNA in the P-site ${ }^{21}$. The most stable binding was observed using RNCs in the presence of ADPNP and the antibiotic neomycin ${ }^{1}$ (Fig. 2a), whereas empty 80 S ribosomes or the presence of ADP (Supplementary Fig. 5) showed weaker binding. As suggested before, this indicates that eEF3 binds to post-state ribosomes, and that ATP hydrolysis is required for eEF3 dissociation from the ribosome $e^{4}$.

Cryo-EM and single-particle three-dimensional reconstruction of the RNC-eEF3 complex shows the typical appearance of a programmed yeast ribosome (Fig. 2b) with a P-site tRNA at a resolution of $9.9 \AA$. Additional density (Supplementary Fig. 6) is present for
eEF3, and consists of two large domains bridging the central protuberance of the $60 \mathrm{~S}$ and the head of the $40 \mathrm{~S}$ ribosomal subunit, respectively. In agreement with binding experiments ${ }^{2,3,22}$, the density of eEF3 could be unambiguously assigned to the HEAT and ABC domains of eEF3, respectively (Fig. 2b).

\section{Ribosomal binding site and conformation of eEF3}

The ribosomal binding site for eEF3 is new and completely separated from the binding site for the canonical factors eEF1A and eEF2 (Fig. 2c). The site involves proteins and ribosomal RNA in both the $40 \mathrm{~S}$ and 60S ribosomal subunit (Fig. 2d; Supplementary Table 2). Non-overlapping binding sites explain how eEF3 and eEF1A can bind simultaneously ${ }^{1}$, and confirm that the competition between eEF3 and eEF2 must be a result of different requirements regarding the conformation of the ribosome ${ }^{1,4}$. Here, eEF3 binds to a post-state ribosome, whereas eEF2 binds to the pre state ${ }^{8,23}$. The structure of the eEF2-ribosome complex (stabilized by the antibiotic sordarin) indeed reveals a rotated conformation of the head of the $40 \mathrm{~S}$ subunit ${ }^{8,23}$, which would hardly allow the binding of eEF3 in the observed conformation.

For building a ribosome-bound eEF3 model (Supplementary Methods), we tried to fit models for eEF3 with the ABC tandem in the ATP state (closed conformation) and ADP state (open conformation). Cross-correlation indicated that eEF3 was indeed present in the ATP state (cross-correlation of 0.78 for ATP versus 0.61 for ADP). The chromodomain had to be moved and rotated relative to its
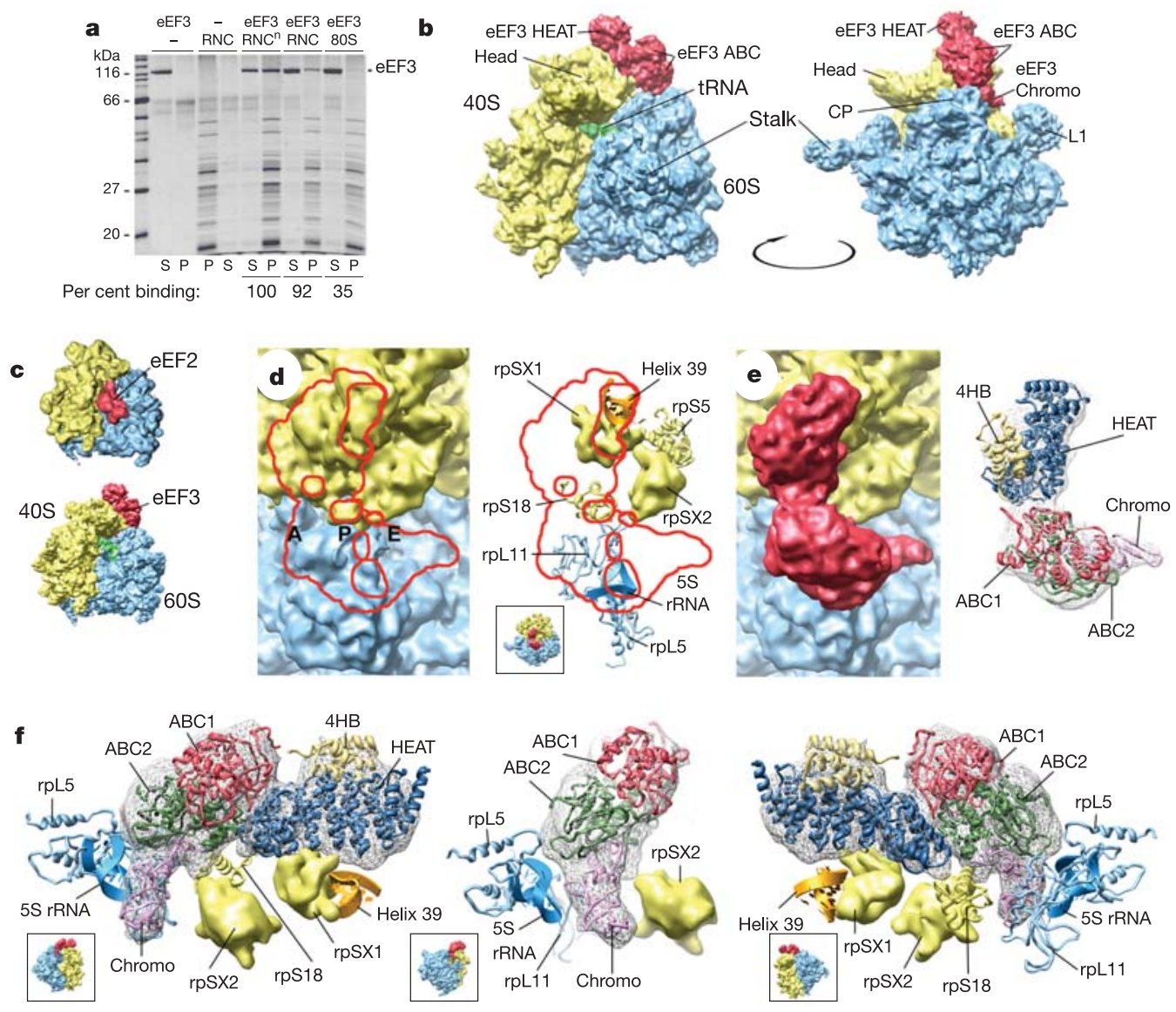

Figure 2 | Reconstitution and cryo-EM structure of the eEF3-ribosome complex. a, Binding assay using RNCs or empty $80 \mathrm{~S}$ ribosomes with eEF3 in the presence of ADPNP, and neomycin (n) where indicated. $S$, supernatant; $\mathrm{P}$, pellet. $\mathbf{b}$, Cryo-EM reconstruction of the eEF3-RNC complex. The right view is rotated by $90^{\circ}$ (arrow). CP, central protuberance. c, Comparison of the cryo-EM map of the 80S-eEF2-sordarin complex ${ }^{23}$ (top) and the eEF3-RNC complex (bottom) reveals different binding sites. d, Top view (see inset) showing the binding site of eEF3 contoured in red on the ribosomal density (left), and with molecular models of ribosomal components (right). 40S components are shown in yellow, $60 \mathrm{~S}$ components in blue. Unknown ribosomal proteins (rp) termed rpSX1 and rpSX2 are represented as electron densities. A-, P- and E-sites are indicated by A, P and E. e, Density and molecular model of eEF3-ATP on the ribosome. f, Molecular contacts of eEF3 with ribosomal components. 
position in the crystal structure. The HEAT domain was subdivided into two blocks consisting of 5 and 3 repeats (residues 1-200 and 201-333, respectively), which were docked together with the $4 \mathrm{HB}$ domain as individual rigid bodies. Notably, the ribosome-bound conformation of eEF3 (Fig. 1d, right panel) fitted neither the pH 7.2 SAXS scattering curve (Supplementary Fig. 4 and Supplementary Note 1) nor the ATP model (Fig. 1d, middle panel), which suggests that a large conformational change takes place on ribosome binding. This is also observed with other translation factors, such as eEF2 ${ }^{23}$ and RF2, on ribosome binding. Compared with the ATP-state model, the first five HEAT repeats rotate by $160^{\circ}$ in the cryo-EM conformation of eEF3 (Fig. 1d).

The ABC2 domain is positioned on the central protuberance of the large ribosomal subunit, whereas $\mathrm{ABC} 1$ does not interact with the ribosome; the chromodomain points towards the ribosomal L1 stalk and is juxtaposed to the E-site (Fig. 2d-f). The ABC2 domain and the chromodomain are anchored to the 60S subunit involving $5 \mathrm{~S}$ rRNA, rpL11 (known as L5p in Escherichia coli) and rpL5 (L18p in E. coli) (Supplementary Table 2). Contacts of ABC2 are established between the residues of the first two ABC2 $\beta$-strands and a highly conserved region preceding the first $\beta$-strand (Supplementary Fig. 1). In the chromodomain, three distinct regions interact with both the 60S and the 40S subunit. Residues 802-808 (representing the Sac7 homology domain, which is known to bind $\mathrm{DNA}^{24}$ ) contact the 5S rRNA and rpL11 in the 60S subunit. Two linker regions connecting the chromodomain with $\mathrm{ABC} 2$ interact with the small ribosomal subunit via $\mathrm{rpS18}$ (S13p in E. coli) and an as yet unassigned density (rpSX2) that is likely to correspond to the N-terminal extension of rpS5 (Fig. 2d-f).

The HEAT domain contacts the head of the 40S subunit via the loops connecting the helices of the HEAT repeats. In agreement with binding studies ${ }^{2}$, this interaction involves the tip of helix 39 of the $18 \mathrm{~S}$ rRNA, an unknown ribosomal protein (here termed rpSX1) and rpS18 (Supplementary Table 2; Fig. 2f). HEAT repeats active in RNA binding have only been observed for the Ro autoantigen, where the helix-connecting loops contact single-stranded RNA via basic amino-acid residues ${ }^{25}$. Likewise, all of the contacts formed between the HEAT domain of eEF3 and ribosomal RNA (and also the ribosomal proteins) involve loops.

\section{Involvement of eEF3 in tRNA release}

The question remains of how eEF3 acts on the ribosome as a dynamic $\mathrm{ABC}$ protein and whether this action can be related to its suggested function as an E-site factor. It is intriguing that the chromodomain of eEF3 is positioned in the immediate proximity of the ribosomal E-site where it is capable of influencing the head of the 40S subunit and the L1 stalk, both of which contribute to the affinity of tRNA for the E-site $e^{23,26,27}$. It is very likely that a conformational switch of the chromodomain occurs on ribosome binding. Such a switch would correlate well with the movement of the L1 stalk from an E-sitesecluding 'in' position to an 'open' position, which would, thus, unlock the E-site to allow release of the tRNA (Fig. 3). An important function of the chromodomain is further supported by its placement between the Q-loop and the signature motif. In BtuCD the corresponding region interacts with the transmembrane domain ${ }^{15}$, and in $\operatorname{Rad} 50^{18}$ this region harbours the coiled-coil forming the ring around two strands of DNA, both of which undergo conformational changes. Likewise, ATP binding and hydrolysis by eEF3 might induce a change in the relative orientation of the two subdomains in $\mathrm{ABC} 2$, and thereby change the position of the chromodomain. We propose that this could, in turn, influence the conformation of the L1 stalk.

\section{Conclusions}

Our findings demonstrate conformational flexibility of eEF3 that is likely to be used for its function. In the eEF3-ADPNP conformation bound to the $80 \mathrm{~S}$ ribosome (Fig. 1d), the two ABC domains dimerize, with the ABC tandem being present in the ATP-bound closed dimer

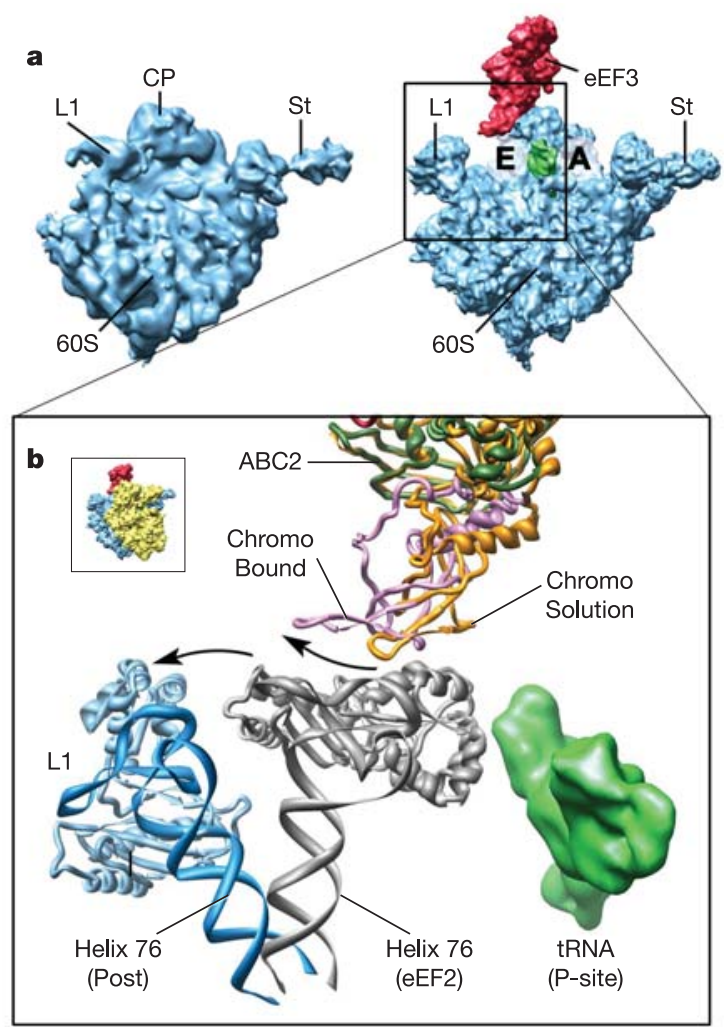

Figure 3 | Dynamic behaviour of eEF3 and the 80 S ribosome. a, Position of eEF3 with respect to the ribosomal $\mathrm{E}$ - and $\mathrm{A}$-sites (indicated by $\mathrm{E}$ and $\mathrm{A}$ ), and to the dynamic L1 stalk (St) in the 'in' and 'out' position as observed in the 80S-eEF2-sordarin complex (left) and the eEF3-RNC complex (right), respectively. b, Movement of the chromodomain of eEF3 and the L1 stalk of the $60 \mathrm{~S}$ subunit. Arrows indicate the movements of the L1 stalk from the eEF2 state (grey) to the post state (blue), and the switch of the chromodomain from a solution-state model (yellow) to the observed ribosome-bound (ATP) state (magenta).

state observed in the ATP-bound structures of Rad50 and MJ0796. Compared with our solution-state model, a large reorientation of the HEAT and $4 \mathrm{HB}$ domains relative to ABC1 has occurred (Fig. 1d). So far, structures of homodimeric $\mathrm{ABC}$ proteins, except for the lowresolution structures of $\mathrm{MsbA}^{17,28}$, have indicated only relatively small differences between the open and closed dimeric states. The crystal structure of eEF3 demonstrates that the nucleotide-binding domains of twin-cassette $\mathrm{ABC}$ proteins may undergo very large intramolecular conformational changes. In eEF3, this could be required especially during binding and release from the ribosome. The SAXS data collected at $\mathrm{pH} 7.2$ showed that the ABC1-ABC2 domain interface most likely pre-forms in solution. The weak intrinsic ribosome-independent ATPase activity ${ }^{29}$ suggests that the $\mathrm{ABC}$ tandem is somewhat open in solution. This is then likely to rearrange to a tighter tandem with higher ATPase activity on ribosome binding, in agreement with the 36-fold stimulation of eEF3 ATPase activity by the ribosome ${ }^{29}$.

We suggest that the chromodomain stabilizes the L1 stalk in the 'out' position (Fig. 3b). Rearrangement of L1 is a prerequisite for the release of the tRNA from the E-site, as this site is secluded with L1 in the 'in' position ${ }^{10,23}$. Therefore, one aspect of eEF3 function may be the opening of the E-site by moving the L1 stalk. Notably, the largest movements of the L1 stalk have been observed in S. cerevisiae ${ }^{23}$, an organism-as are all other fungi-strictly dependent on eEF3 activity. An essential function of the chromodomain on the ribosome explains why proteolytic degradation of this domain preserves the intrinsic ATPase activity but destroys the ribosome-dependent stimulation $^{22}$. 


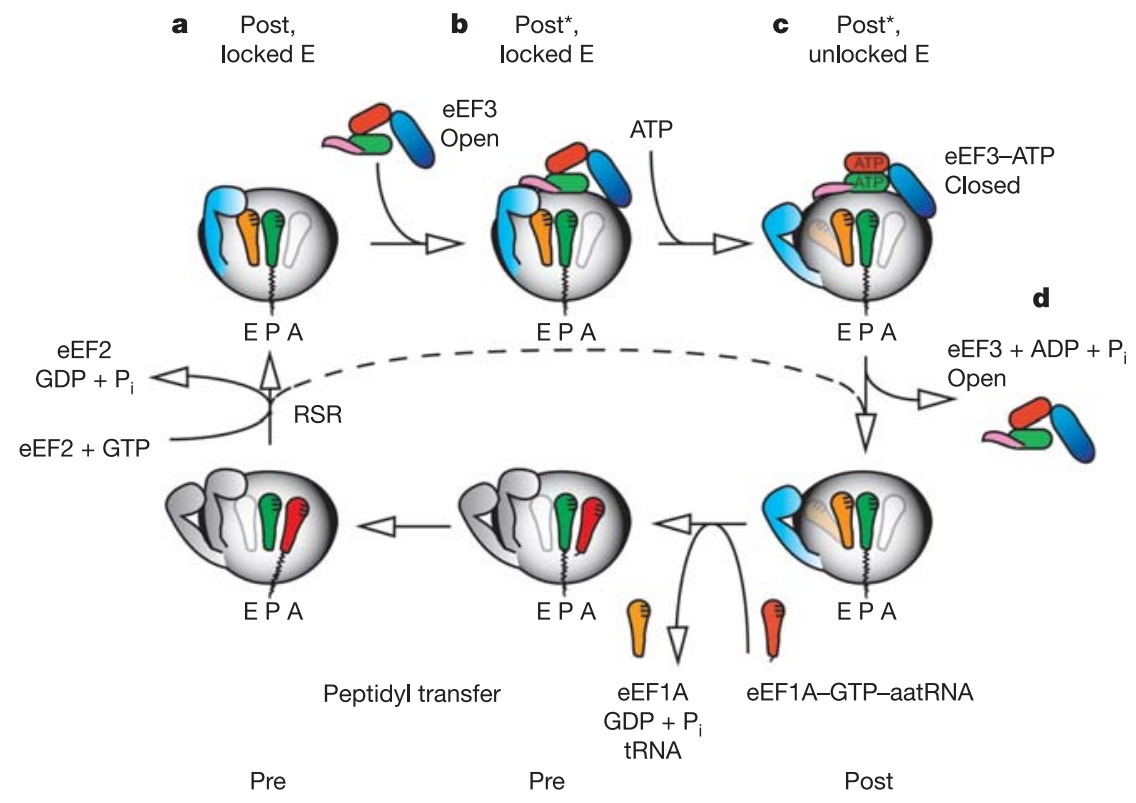

Figure $4 \mid$ Model of the role of eEF3 in the fungal elongation cycle. a, The post-state ribosome with a locked E-site tRNA owing to the L1 stalk in the 'in' position and the conformation of the $40 \mathrm{~S}$ head (Post, locked E). b, Hypothetical initial interaction of eEF3 in the open tandem or intermediate conformation (Post ${ }^{*}$, locked E). c, Ribosome interaction triggers the ATP-dependent closed tandem formation and high-affinity ribosome binding by eEF3, as observed by cryo-EM (Post $\left.{ }^{\star}\right)$.

The conformational switch of eEF3 could also lead to a change of the $40 \mathrm{~S}$ head conformation via the HEAT domain, resulting in altered properties of the E-site $e^{23,26,27}$. This would also follow the general principle of $A B C$ transporters, where the rearrangement of the two $\mathrm{ABC}$ domains between an open and a closed dimer state is used for the rearrangement of the domains connected to them. eEF3 itself is partially closing the E-site and stalls the head of the $40 \mathrm{~S}$ subunit in its position. Hence, it is likely that tRNA release is dependent on the dissociation of eEF3 and release of the stalled conformation of the $40 S$ subunit head. This would explain why efficient chasing from the E-site with cognate deacyl-tRNA is dependent on ATP hydrolysis by $\mathrm{eEF}^{4}$, which would trigger the dissociation of eEF3 from the ribosome.

Following the switch model for $\mathrm{ABC}$ transporters, in which substrate binding triggers ATP binding ${ }^{30}$, eEF3 binds to the poststate ribosomes also in the presence of ADP (Supplementary Fig. 5), indicative of the open conformation of the $\mathrm{ABC}$ tandem. On this basis, we suggest a model describing the role of eEF3 in the fungal translation cycle, which is expanded by fungi-specific post states of the ribosome (Fig. 4). (1) After eEF2-induced tRNA translocation, the conformation of the $40 \mathrm{~S}$ head and of the L1 stalk in the 'in' position maintains the E-site tRNA as secluded (Fig. 4, "Post, locked E"). (2) Initial weak interaction of eEF3 with the ribosome may occur in the open $\mathrm{ABC}$ tandem, or an intermediate conformation of the $\mathrm{ABC}$ domains, without inducing changes in the ribosome ("Post', locked E"). (3) The interaction with the ribosome may trigger the ATP-dependent closed tandem formation and high-affinity ribosome binding by eEF3 in the conformation observed by cryo-EM. A conformational switch of the eEF3 chromodomain may stabilize the L1 stalk in the 'out' position, thereby opening the E-site ("Post*, unlocked E"). Now, eEF3 itself partially closes the E-site and fixes the 40S head. (4) The closed tandem elicits ATP hydrolysis, causing the dissociation of eEF3 from the ribosome. This unlocks the $40 \mathrm{~S}$ head and results in an open E-site from which the tRNA can be released, probably owing to an increased off-rate ("Post"). Now, aminoacyltRNA-eEF1A-GTP can deliver the next aminoacyl-tRNA to the

A conformational switch of the chromodomain stabilizes the L1 stalk in the 'out' position (unlocked E). d, ATP hydrolysis of the closed tandem results in the dissociation of eEF3, E-site opening, and unlocking of the 40S head (Post). Now, eEF1A-GTP-aminoacyl-tRNA can bind and the E-site deacyltRNA is released. ATP hydrolysis by eEF3, tRNA release, and A-site loading by eEF1A may take place as a joint event. aatRNA, aminoacyl-tRNA. RSR, ratchet-like subunit rearrangement.

A-site. According to the allosteric three-site model ${ }^{4}$, a closed E-site preventing release of deacyl-tRNA would interfere with A-site binding. As eEF3 and eEF1A can bind simultaneously to the ribosome, ATP hydrolysis by eEF3, tRNA release, and A-site loading by eEF1A may take place as a joint event.

Taken together, we have provided the structural basis for understanding the essential function of eEF3, which will be instrumental for the future dissection of the exact molecular mechanism of this unique fungal factor, and, in addition, may be used for rational drug design.

\section{METHODS}

An initial polyalanine model was built into the $3.5 \AA$ resolution density map of eEF3-ADP ${ }^{12}$. This model was used for molecular replacement with the $2.4 \AA$ selenomethionine data set. Phase combination allowed the construction of a new polyalanine model by RESOLVE ${ }^{31}$, rebuilding in $\mathrm{O}^{32}$, and refinement with $\mathrm{CNS}^{33}$ (Supplementary Table 1). Crystals of apo eEF3 and eEF3-ADPNP were obtained using the same approach as eEF3-ADP ${ }^{12}$ by co-crystallization in the presence of $45 \%(\mathrm{w} / \mathrm{v})$ ammonium sulphate at $\mathrm{pH} 5.2$, and data processed with $\mathrm{XDS}^{34}$ (Supplementary Table 1). The eEF3-ADP structure was used for rigid-body refinement against these data, and the resulting model was manually refitted in $\mathrm{O}$ and refined in CNS (Supplementary Table 1).

The SAXS measurements were performed ${ }^{35}$ at $\mathrm{pH} 5.2$ or $\mathrm{pH} 7.2$, and $1 \mathrm{mM}$ ADP/ATP when present. Simulated SAXS scattering curves were calculated with $\mathrm{CRYSOL}^{36}$, and experimental molecular envelopes with GASBOR ${ }^{37}$.

The 80 S ribosomes and RNCs were purified as described before ${ }^{21,38,39}$. eEF3$\mathrm{Xa}-\mathrm{His}_{10}$ was overexpressed in yeast and affinity-purified. $80 \mathrm{~S}$ ribosome-eEF3 complexes were reconstituted and applied on carbon-coated holey grids ${ }^{40}$ in the presence of Sec6 $1^{21}$, visualized on a Polara cryo-microscope, and processed using SPIDER $^{41}$. The data set was sorted according to empty ${ }^{38}$ or programmed ${ }^{21}$ conformational state, followed by sorting according to the presence of eEF3. A total of 37,700 particles were used for the final reconstruction at a resolution of $9.9 \AA(6.2 \AA)$ based on Fourier shell correlation of masked volumes at $0.5(3 \sigma)$. The map is shown without the Sec61 density in Figs 2 and 3 for better clarity (see Supplementary Fig. 6a for the complete map).

Docking of X-ray structures and molecular models of eEF3 $3^{42,43}$ and ribosomal proteins/RNA ${ }^{44}$ was done with IRIS Explorer, SPIDER ${ }^{41}, \mathrm{O}^{32}$ and ERNA-3D ${ }^{45}$. Using the program Situs ${ }^{46}$ for docking of MalK-based ${ }^{47}$ ATP and ADP models for 
eEF3 (cross-correlation of 0.7 and 0.62 , respectively) confirmed our results with models based on MJ0796 ${ }^{42}$ and RLI ${ }^{43}$. Figures were produced with PYMOL ${ }^{48}$ and USFC Chimera $^{49}$.

\section{Received 10 October 2005; accepted 3 August 2006.}

\section{Published online 23 August 2006.}

1. Kovalchuke, O., Kambampati, R., Pladies, E. \& Chakraburtty, K. Competition and cooperation among yeast elongation factors. Eur. J. Biochem. 258 986-993 (1998)

2. Gontarek, R. R., Li, H., Nurse, K. \& Prescott, C. D. The N terminus of eukaryotic translation elongation factor 3 interacts with 18S rRNA and 805 ribosomes. J. Biol. Chem. 273, 10249-10252 (1998)

3. Kambampati, R. et al. Limited proteolysis of yeast elongation factor 3. Sequence and location of the subdomains. J. Biol. Chem. 275, 16963-16968 (2000).

4. Triana-Alonso, F. J., Chakraburtty, K. \& Nierhaus, K. H. The elongation factor 3 unique in higher fungi and essential for protein biosynthesis is an $\mathrm{E}$ site factor. J. Biol. Chem. 270, 20473-20478 (1995).

5. Kamath, A. \& Chakraburtty, K. Role of yeast elongation factor 3 in the elongation cycle. J. Biol. Chem. 264, 15423-15428 (1989).

6. Jones, P. M. \& George, A. M. Mechanism of ABC transporters: a molecular dynamics simulation of a well characterized nucleotide-binding subunit. Proc. Natl Acad. Sci. USA 99, 12639-12644 (2002).

7. Frank, J. \& Agrawal, R. K. A ratchet-like inter-subunit reorganization of the ribosome during translocation. Nature 406, 318-322 (2000).

8. Gomez-Lorenzo, M. G. et al. Three-dimensional cryo-electron microscopy localization of EF2 in the Saccharomyces cerevisiae 80 S ribosome at $17.5 \AA$ resolution. EMBO J. 19, 2710-2718 (2000).

9. Valle, M. et al. Locking and unlocking of ribosomal motions. Cell 114, 123-134 (2003).

10. Harms, J. et al. High resolution structure of the large ribosomal subunit from a mesophilic eubacterium. Cell 107, 679-688 (2001)

11. Morgan, D. G., Menetret, J. F., Neuhof, A., Rapoport, T. A. \& Akey, C. W. Structure of the mammalian ribosome-channel complex at $17 \AA$ resolution. J. Mol. Biol. 324, 871-886 (2002).

12. Andersen, C. F. et al. Purification and crystallization of the yeast translation elongation factor eEF3. Acta Crystallogr. D 60, 1304-1307 (2004).

13. Andrade, M. A., Petosa, C., O'Donoghue, S. I., Muller, C. W. \& Bork, P. Comparison of ARM and HEAT protein repeats. J. Mol. Biol. 309, 1-18 (2001).

14. Groves, M. R., Hanlon, N., Turowski, P., Hemmings, B. A. \& Barford, D. The structure of the protein phosphatase 2A PR65/A subunit reveals the conformation of its 15 tandemly repeated HEAT motifs. Cell 96, 99-110 (1999).

15. Locher, K. P., Lee, A. T. \& Rees, D. C. The E. coli BtuCD structure: a framework for ABC transporter architecture and mechanism. Science 296, 1091-1098 (2002).

16. Smith, P. C. et al. ATP binding to the motor domain from an $A B C$ transporter drives formation of a nucleotide sandwich dimer. Mol. Cell 10, 139-149 (2002).

17. Chang, G. \& Roth, C. B. Structure of MsbA from E. coli: a homolog of the multidrug resistance ATP binding cassette (ABC) transporters. Science 293, 1793-1800 (2001).

18. Hopfner, K. P. et al. Structural biology of Rad50 ATPase: ATP-driven conformational control in DNA double-strand break repair and the ABCATPase superfamily. Cell 101, 789-800 (2000).

19. Holm, L. \& Sander, C. Mapping the protein universe. Science 273, 595-603 (1996).

20. Brehm, A., Tufteland, K. R., Aasland, R. \& Becker, P. B. The many colours of chromodomains. Bioessays 26, 133-140 (2004).

21. Beckmann, R. et al. Architecture of the protein-conducting channel associated with the translating 80 S ribosome. Cell 107, 361-372 (2001).

22. Kambampati, R. \& Chakraburtty, K. Functional subdomains of yeast elongation factor 3. Localization of ribosome-binding domain. J. Biol. Chem. 272, 6377-6381 (1997).

23. Spahn, C. M. et al. Domain movements of elongation factor eEF2 and the eukaryotic 80 S ribosome facilitate tRNA translocation. EMBO J. 23, 1008-1019 (2004).

24. Robinson, $\mathrm{H}$. et al. The hyperthermophile chromosomal protein Sac7d sharply kinks DNA. Nature 392, 202-205 (1998).

25. Stein, A. J., Fuchs, G., Fu, C., Wolin, S. L. \& Reinisch, K. M. Structural insights into RNA quality control: the Ro autoantigen binds misfolded RNAs via its central cavity. Cell 121, 529-539 (2005).

26. Yusupov, M. M. et al. Crystal structure of the ribosome at $5.5 \AA$ resolution. Science 292, 883-896 (2001).

27. Ogle, J. M. \& Ramakrishnan, V. Structural insights into translational fidelity. Annu. Rev. Biochem. 74, 129-177 (2005).

28. Reyes, C. L. \& Chang, G. Structure of the ABC transporter MsbA in complex with ADP·vanadate and lipopolysaccharide. Science 308, 1028-1031 (2005).
29. Sarthy, A. V. et al. High-level overexpression of yeast elongation factor 3 and detailed kinetic analysis using a coupled spectrophotometric assay. Anal. Biochem. 254, 288-290 (1997).

30. Higgins, C. F. \& Linton, K. J. The ATP switch model for ABC transporters Nature Struct. Mol. Biol. 11, 918-926 (2004).

31. Terwilliger, T. SOLVE and RESOLVE: automated structure solution, density modification and model building. J. Synchrotron Radiat. 11, 49-52 (2004).

32. Jones, T. A., Cowan, S., Zou, J.-Y. \& Kjeldgaard, M. Improved methods for building protein models in electron density maps and the location of errors in these models. Acta Crystallogr. A 47, 110-119 (1991).

33. Brunger, A. T. et al. Crystallography \& NMR system: a new software suite for macromolecular structure determination. Acta Crystallogr. D 54, 905-921 (1998).

34. Kabsch, W. in International Tables for Crystallography (ed. Arnold, E.) Ch. 25.2.9 (Kluwer Academic, Dordrecht, 2001).

35. Pedersen, J. S. A flux- and background-optimized version of the NanoSTAR small-angle X-ray scattering camera for solution scattering. J. Appl. Cryst. 37, 369-380 (2004)

36. Svergun, D. I., Barberato, C. \& Koch, M. H. J. CRYSOL-a program to evaluate $\mathrm{X}$-ray solution scattering of biological macromolecules from atomic coordinates. J. Appl. Cryst. 28, 768-773 (1995)

37. Svergun, D. I., Petoukhov, M. V. \& Koch, M. H. J. Determination of domain structure of proteins from X-ray solution scattering. Biophys. J. 80, 2946-2953 (2001).

38. Beckmann, R. et al. Alignment of conduits for the nascent polypeptide chain in the ribosome-Sec61 complex. Science 278, 2123-2126 (1997).

39. Halic, M. et al. Structure of the signal recognition particle interacting with the elongation-arrested ribosome. Nature 427, 808-814 (2004).

40. Wagenknecht, T., Grassucci, R. \& Frank, J. Electron microscopy and computer image averaging of ice-embedded large ribosomal subunits from Escherichia coli. J. Mol. Biol. 199, 137-147 (1988)

41. Frank, J. et al. SPIDER and WEB: processing and visualization of images in 3D electron microscopy and related fields. J. Struct. Biol. 116, 190-199 (1996).

42. Yuan, Y. R. et al. The crystal structure of the MJ0796 ATP-binding cassette Implications for the structural consequences of ATP hydrolysis in the active site of an ABC transporter. J. Biol. Chem. 276, 32313-32321 (2001).

43. Karcher, A., Buttner, K., Martens, B., Jansen, R. P. \& Hopfner, K. P. X-ray structure of RLI, an essential twin cassette ABC ATPase involved in ribosome biogenesis and HIV capsid assembly. Structure (Camb.) 13, 649-659 (2005).

44. Spahn, C. M. et al. Structure of the 805 ribosome from Saccharomyces cerevisiae-tRNA-ribosome and subunit-subunit interactions. Cell 107, 373-386 (2001).

45. Mueller, F. et al. Getting closer to an understanding of the three-dimensional structure of ribosomal RNA. Biochem. Cell Biol. 73, 767-773 (1995).

46. Wriggers, W., Milligan, R. A. \& McCammon, J. A. Situs: a package for docking crystal structures into low-resolution maps from electron microscopy. J. Struct. Biol. 125, 185-195 (1999).

47. Lu, G., Westbrooks, J. M., Davidson, A. L. \& Chen, J. ATP hydrolysis is required to reset the ATP-binding cassette dimer into the resting-state conformation. Proc. Natl Acad. Sci. USA 102, 17969-17974 (2005)

48. DeLano, W. L. The PyMOL User's Manual (DeLano Scientific, San Carlos, California, 2002).

49. Pettersen, E. F. et al. UCSF Chimera-a visualization system for exploratory research and analysis. J. Comput. Chem. 25, 1605-1612 (2004).

Supplementary Information is linked to the online version of the paper at www.nature.com/nature.

Acknowledgements G.R.A. was supported by the NIH, HFSP, EU FP5 and SNF. T.G.K. was supported by the NIH and HFSP. We are grateful to I. Kerr and D. Brodersen for discussions, and to L. Van for technical assistance. The work was further supported by grants from the VolkswagenStiftung (to R.B. and C.M.T.S.), the Deutsche Forschungsgemeinschaft (to R.B.), and by the European Union and Senatsverwaltung für Wissenschaft, Forschung und Kultur Berlin (UltraStructureNetwork).

Author Information Coordinates and structure factors for the crystal structures have been deposited in the RCSB Protein Data Bank under accession numbers 2IX3, 2IW3 and 2IWH; the electron microscopy (EM) map has been deposited in the EMBL-European Bioinformatics Institute EM Data Bank under accession number EMD-1233 and coordinates of EM-based models in the RCSB Protein Data Bank under accession number 2IX8. Reprints and permissions information is available at www.nature.com/reprints. The authors declare no competing financial interests. Correspondence and requests for materials should be addressed to R.B. (beckmann@Imb.uni-muenchen.de) and G.R.A. (gra@mb.au.dk). 